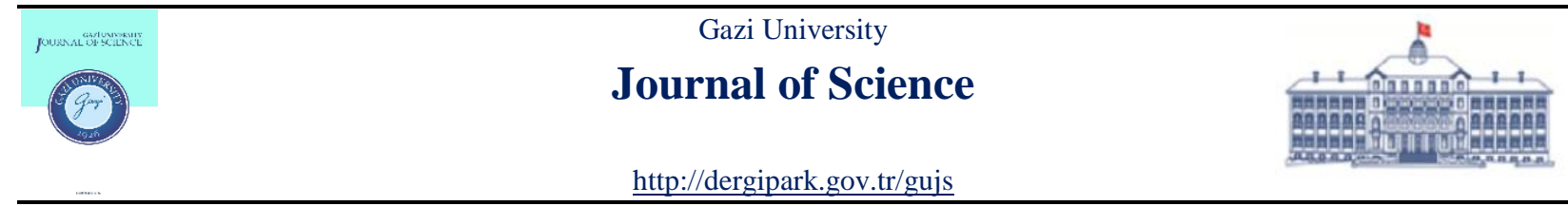

\title{
A Novel Application of Senstivity Factor (Ptdf) for Transmission Pricing Evaluation in a Deregulated Power Market
}

\author{
Amit Kumar SINGH ${ }^{1, *(1)}$, Cuneyt Suheyl OZVEREN ${ }^{2}$ (i⿱一⿻丿口乚 \\ ${ }^{I}$ School of Electronics and Electrical Engineering, Lovely Professional University, Jalandhar, Punjab (India) \\ ${ }^{2}$ School of Engineering and Technology, Abertay University, Dundee, Scotland (U.K.)
}

\section{Highlights}

- Application of sensitivity factor for Transmission pricing evaluation

- Evaluation of Load and Generator contribution for Power flow.

- Determination of different pricing methods with the proposed PTDF method.

- Comparative analysis of proposed PTDF method with different pricing methods.

\section{Article Info}

Received: $15 / 09 / 2018$

Accepted: 30/08/2019

\section{Keywords}

Transmission Pricing Load Flow Analysis

Distribution Factors

\begin{abstract}
The selection and application of pricing method is generally influenced by the concern of investors and participators. In this paper, the results of the broad assessment of the transmission pricing and the summary of potential key methodologies to explore, are listed and reported. This paper outlines a "hybrid analysis and decision-making framework" for transmission costing and pricing. The proposed hybrid framework facilitates a comparative costing approach of several widely used well-known cost allocation algorithms based online flows and a "generator contributions and transmission pricing" approach with the proposed application of power transfer distribution factors (PTDF) method. The paper reports the results of IEEE 30-bus system case study that demonstrates the effectiveness of the framework. In this paper, a full AC load flow is utilized to determine the line capacity-use, as it is evaluated by the quantity of power transmitted. This approach also allows to look into the effects of reactive power.
\end{abstract}

\section{INTRODUCTION}

Electrical power system was vertically integrated earlier, now it has been deregulated and Generation, Transmission \& Distribution are separate entities. The breakup has been encouraged by tremendous benefits, resulting in competition leading to economy and better consumer services [1]. In the competitive electricity system, power industry wants to create returns to reimburse the possessors of transmission resources and to generate the economic indications for competent venture in transmission and for the resourceful place of new generation plant and loads. This can be achieved by choosing optimal pricing method for transmission of power. The embedded costs [2] of the transmission pact are usually the largest factor of total transaction cost. The embedded cost can be considered as association of two parts, apportionment related to capacity use and reliability benefits. For apportionment based on capacity use AC power flow algorithm [3] can be used. Many pricing schemes has been developed, depending upon the application. Some are based upon uniform pricing for all the consumers (Spot Pricing) and some are based upon the location of consumers (Locational Marginal Pricing) [4]. Further for determining the transmission pricing, load following based methods and generations based methods have been proposed in few literatures which used correlation factors and linear regression techniques [5]. Considering most of the available transmission costing methods, an evaluation and comparison has been done with respect to the proposed application of PTDF method in this paper. Where it is found that the proposed method can reasonably generate better revenues as compare to other methods, which can be utilized for future expansion and quality improvement of transmission system. 


\section{BACKGROUND}

Transmission pricing is among the crucial components in a competitive electricity market. The two extreme approaches for transmission pricing in India are spot pricing approach and MW mile pricing [6]. In which it has seen that for allocation based on capacity use, an AC power flow algorithm using MW mile method can be implemented. This method will apportion the burdens for each wheeling participants considering the use of current transmission conveniences. It's easily understandable and can also implement for real power systems. The effect on system reliability is influenced by selection of pricing methods and MW mile method provides improved system reliability as compared to other methods, because of innate mechanism of discouraging long distance transactions.

The main function of an electrical power system is to deliver trustworthy and uninterrupted electrical energy to placate system demand. The three core constituents of power system are: generation, transmission and distribution systems. The generation systems generates power and transmission systems transport the generated power to distribution systems for satisfying loads. The generation systems along with transmission systems are generally called the composite system or the bulk power system [7].

\section{ESTABLISHED PRICING METHODS}

Most current operational models in deregulated or vertically structured Electricity Supply Industries are based on the Transmission and Distribution Services being provided as a separate item. Although the primary functions of transmission and distribution lines are to transfer power from generation to demand, they also are vital in terms of network security and therefore supply. As the availability or otherwise of a line effects power/load flows in the network and therefore whether a transaction over a line or a route can happen or not.

\subsection{Postage Stamp Method}

Under this practice the transmission users are billed on the basis of average embedded cost and the level of the transmitted power for a particular transaction [8]. However, this method is simple but the actual power flow in the network is ignored and is evaluated using Equation (1)

$\mathrm{RT}=\mathrm{TC} *(\mathrm{Pt} / \mathrm{Ppeak})$

where, RT: Price of transmission for transaction' $T$ '

TC: Transmission Charges

Pt: Transaction ' $\mathrm{T}$ ' load at peak load time in MW

Ppeak: Peak load in MW.

The main disadvantage of this method is that it does not differentiate the near or far distant consumers and presents an inaccurate charging process.

\subsection{MW-Mile Method}

This method deals with the burdens on the consumer, based upon the extent of the use of transacted power during the transaction [2]. The method overcomes the drawbacks of the other mentioned methods and can be evaluated using Equation (2).

There are three approaches of MW-Mile method.

$\mathrm{TCk}=\sum($ Lij Fij Pijk $) /$ Pijmax $\quad$,for i=1-n

where, TCK :Transmission price for transaction $\mathrm{k}$

Lij : Line Length i-j

Fij : Power Cost in line i-j 
Pijk : Flow of Power in line i-j for transaction k.

Pijmax : Thermal limit of line i-j.

Based on transaction ' $\mathrm{k}$ ' the change in line power flow is considered using three approaches.

(a) Absolute MW-Mile

In this method the direction of power flow is not considered in the transmission network for calculating the prices of transmission. The power flow due to customer ' $k$ ' on the line ' $i$ ' is considered with a condition given by Equation (3),

$\mathrm{Pik}=|\mathrm{Pik}|$ for direct and reverse power flows.

(b) Reverse MW-Mile

In this method the reverse direction power flow is also considered in pricing and the price is based upon the total flow. It is noted that the power flow in the reverse direction lowers the load on the transmission line and hence lowers the chances of congestion in the system. The power flow due to customer ' $\mathrm{k}$ ' on the line ' $\mathrm{i}$ ' is considered with a condition which is given in Equations (4) and (5),

Pik $=$ Positive $(+)$ for direct power flows,

$\&$ Pik $=$ Negative $(-)$ for reverse power flows .

(c) Dominant MW-Mile

This method is a combination of absolute and reverse MW mile method. In this method the pricing for customers is performed using direct power flow caused in the transmission line by the customers, without considering the reverse line flow and hence, the customers are not charged for any reverse power flows. In this method the power flow due to customer ' $k$ ' on the line ' $i$ ' is considered with a condition given by Equations (6) and (7),

Pik $=\mid$ Pik $\mid$ for direct power flows

Or, Pik $=0$ for reverse power flows.

\subsection{Bialek's Tracing Method}

The power lines are considered as loss-less for tracing method i.e. the flow of electrical power is same from sending to receiving end [9]. The basic practice to have power flow without losses is by taking a mean of the power flow between both terminals of the line and half of the power lost in each side of the power injected is further added. The flow of power in the transmission line considering generators and loads are evaluated using upstream and downstream processes.

(a) Upstream algorithm:

In this algorithm the overall power flow in the direction of the node is considered and expressed in Equation (8) as,

$P_{i}^{g}=\sum_{j \in \alpha_{i}^{(u)}}\left|P_{i j}^{g}\right|+P_{G i}$

where, $\mathrm{i}=1,2,3 \ldots \mathrm{n}$

$\propto_{i}^{(u)}$, all nodes supplying power to nodes directly

$P_{i}{ }^{g}$, overall unknown Power Flowing through line 'i'

$\left|P_{i j}{ }^{g}\right|$, total unknown Power Flowing from node $\mathrm{j}$ to $\mathrm{i}$

$P_{G i}$, power generation at node i. 
As, $\left|P_{i j}{ }^{g}\right|=\left|P_{j i}{ }^{g}\right|$ considering loss less lines,

$\left|P_{i j}{ }^{g}\right|$ is written as $\left(P_{j i}^{g} / P_{j}{ }^{g}\right) * P_{j}{ }^{g}$, considering small transmission losses then $P_{j i}{ }^{g} / P_{j}{ }^{g} \approx P_{j i} / P_{j}$ where, ' $P_{j i}$ ' is the value of Power Flow from node ' $\mathrm{j}$ ' in line ' $\mathrm{ji}$ ' and ' $P$ ' $j$ ' is the actual flow at node ' $\mathrm{j}$ '.

The conclusion is that the overall distributed power flow at any node is analogous to the actual Power Flow through that node, and is expressed as Equation (9),

$P_{i}-\sum_{j \in \alpha_{i}^{(u)}} \frac{\left|P_{j i}\right|}{P_{j}} * P_{j}^{g}=P_{G i}$

or, $\quad A_{u} P_{\text {gross }}=P_{G}$

where, $A_{u}$ is the upstream distribution matrix shown in Equation (11), $P_{\text {gross }}$ is unknown gross nodal Power Flow and $P_{G}$ is nodal generation.

$\left[A_{u}\right]_{i j}=\left\{\begin{array}{cc}1 & \text { for } i=j \\ -\frac{\left|P_{j i}\right|}{P_{j}} & \text { for } j \in \propto_{i}^{(u)} \\ 0 & \text { otherwiswe }\end{array}\right.$

or, $P_{\text {gross }}=A_{u}{ }^{-1} P_{G}$

$P_{i}^{g}=\sum_{k=1}^{n}\left[A_{u}^{-1}\right] P_{G k}$, is the ' $i$ 'th element of total power.

The absolute equation for outflow in line 'ij' from 'ith' node considering the principle of proportionate sharing is shown in Equation (13)

$P_{i}^{g}=\frac{P_{j i} i^{g}}{P_{j}{ }^{g}} * P_{j}^{g}=\frac{P_{j i}{ }^{g}}{P_{j}{ }^{g}} \sum_{k=1}^{n}\left[A_{u}^{-1}\right] P_{G k}$

$P_{i}^{g}=\sum_{k=1}^{n} D_{i j, k}^{g} P_{G k}$ for $j \in \propto_{i}^{d}$

where, $\propto_{i}^{d}$ is the set of nodes supplied from node $i$.

$D_{i j, k}^{g}=\frac{P_{j i}{ }^{g}}{P_{j}{ }^{g}}\left[A_{u}^{-1}\right]$ is the distribution factor.

This distribution factor provides the amount of the generated power from the specific generator for load flow and its sign is positive at all times.

(b) Downstream algorithm:

The downstream algorithm and upstream algorithm are similar at some grounds but the major dissimilarity is that downstream algorithm provides contribution of every load resulting the flow of power in the transmission line where $P_{i}^{l}$ is the summation of all outward flow between load and node ' $\mathrm{i}$ '.

\section{PROPOSED TRANSMISSION PRICING METHOD (PTDF METHOD)}

PTDF is defined as the relative variation in the power flow on a particular branch from bus ' $i$ ' to bus ' $j$ ' due to variation in injected power and corresponding withdrawal at the system swing or slack bus. To determine PTDF the Jacobian matrix is made, which gives the relation of flow of power at both ends of the line with the variation of magnitude and angles of voltage [10]. PTDF's remain moreover constant in the line during different loading conditions. 
This method uses the part of amount of power flows from one node to another in a transmission line [11]. This method considers both D.C. and AC Power flow solutions (becomes ACPTDF). It is applied to calculate the maximum limit of flow in a pair of transactions between end points. This is one of the sensitivity based power flow methods [12-15].

Power Transfer Distribution Factors (PTDF) exhibits linear impact of power transfer i.e. it gives the percentage of power transfer in each transmission line of a power system. It can be calculated using power flow jacobians.

For a transaction 'Pab' among seller and buyer at bus 'a' and bus 'b'. And a line 'l' carrying a fraction of the transaction power which is linked between bus ' $i$ ' and bus ' $j$ '. The change in real power transaction among the above seller and buyer by $\triangle \mathrm{Pab}$, for the change in transmission line quantity $\triangle \mathrm{Pij}$, the PTDF can be expressed using Equation (14) as:

$P T D F_{i j, a b}=\Delta P_{i j} / \Delta P_{a b}$

For power deliveries and for system operations it is important to know that the intensity of current in each branches of the meshed network is inversely proportional to the branch resistance. The PTDF can also be evaluated using physical parameters like reactance $(X)$ or susceptance $(B=1 / X)$ as given by Equation (15):

$P T D F=B^{-1} * B_{f}$

where, $B^{-1}$ : reduced inverse of nodal susceptance matrix

$B_{f}$ : reduced branch Susceptance matrix.

Reduced means, the rows and columns corresponding to a reference node are eliminated.

Using Power Flow Jacobians PTDF can be derived as follows:

Power flow sensitivity and Jacobian of power injection equations is required for PTDF calculation using AC Load Flow. The Jacobian is evaluated using N-R load flow. In polar form the power flow equations is expressed as given by Equations (16) and (17) :

$P_{i}=\sum_{j=1}^{n}\left|V_{i}\right|\left|V_{j}\right|\left|Y_{i j}\right| \cos \left(\theta_{i j}-\delta_{i}+\delta_{j}\right)$

$Q_{i}=\sum_{j=1}^{n}\left|V_{i}\right|\left|V_{j}\right|\left|Y_{i j}\right| \sin \left(\theta_{i j}-\delta_{i}+\delta_{j}\right)$

where,

'n' total buses

$P_{i}$ and $Q_{i}$ real and reactive power injected at 'i'th bus.

$\left|V_{i}\right|,\left|V_{j}\right|$ voltage magnitudes at bus 'i' and ' $\mathrm{j}$ ',

$\delta_{i}, \delta_{j}$ voltage angles at the bus ' $\mathrm{i}$ ' and bus ' $\mathrm{j}$ ', and

$\left|Y_{i j}\right|, \theta_{i j}$ from bus admittance matrix.

The change in power flows at any bus is formulated in terms of Jacobian, using Taylor series expansion, as given by Equation (18):

$\left[\begin{array}{c}\Delta P \\ \Delta Q\end{array}\right]=\left[\begin{array}{ll}J_{1} & J_{2} \\ J_{3} & J_{4}\end{array}\right]\left[\begin{array}{c}\Delta \delta \\ \delta|V|\end{array}\right]$.

The change in the angle and voltage magnitude is determined using Equation (19): 


$$
\left[\begin{array}{c}
\Delta \delta \\
\delta|V|
\end{array}\right]=\left[\begin{array}{cc}
J_{1} & J_{2} \\
J_{3} & J_{4}
\end{array}\right]^{-1}\left[\begin{array}{c}
\Delta P \\
\Delta Q
\end{array}\right]
$$

Bus voltage magnitudes and angles are obtained using N-R load flow analysis. Jacobian and power flow sensitivity [16] are calculated to calculate Power Transfer Distribution Factors. The sensitivity of power flow is evaluated using power flow equations for real power.

The real power flow 'Pij' in a line ' $\mathrm{k}$ ', connected between buses 'i' and 'j', is calculated using Equation (20):

$P_{i j}=V_{i} V_{j} Y_{i j} \cos \left(\theta_{i j}-\delta_{i}+\delta_{j}\right)-V_{i}^{2} Y_{i j} \cos \left(\theta_{i j}\right)$

where, $V_{i}$ and $\delta_{j}$ are the voltage magnitude and angle at bus ' $\mathrm{i}$ '.

$Y_{i j}$ and $\theta_{i j}$ are the magnitude and angle of ijth element of YBus.

Change in real power can be found by using Taylor's series approximation and ignoring higher order terms, as given by Equation (21):

$\Delta P_{i j}=\frac{\partial P_{i j}}{\partial \delta_{i}} \Delta \delta_{i}+\frac{\partial P_{i j}}{\partial \delta_{j}} \Delta \delta_{j}+\frac{\partial P_{i j}}{\partial V_{i}} \Delta V_{i}+\frac{\partial P_{i j}}{\partial V_{j}} \Delta V_{j}$

The sensitivity coefficients can be found using the partial derivatives of real power flow in terms of $\mathrm{J} 1, \mathrm{~J} 2$, $\mathrm{J} 3$ and $\mathrm{J} 4$, w.r.t. variables ' $\delta$ ' and ' $\mathrm{V}$ '.

The power flow equation sensitivity in matrix form can be given by Equation (22):

$\Delta P_{i j}=\left[\frac{\partial P_{i j}}{\partial \delta_{2}}, \ldots, \frac{\partial P_{i j}}{\partial \delta_{n}}, \frac{\partial P_{i j}}{\partial V_{g+1}}, \ldots, \frac{\partial P_{i j}}{\partial V_{n}}\right]\left[\begin{array}{c}\Delta \delta_{i} \\ \cdot \\ \cdot \\ \Delta \delta_{i} \\ \Delta\left|V_{g+1}\right| \\ \cdot \\ \cdot \\ \Delta\left|V_{n}\right|\end{array}\right]$

where, $\left[\frac{\partial P_{i j}}{\partial \delta_{2}}, \ldots . . \frac{\partial P_{i j}}{\partial \delta_{n}}, \frac{\partial P_{i j}}{\partial V_{g+1}}, \ldots . . \frac{\partial P_{i j}}{\partial V_{n}}\right]$ provides the line sensitivity to power flow with respect to angle and magnitude of voltage.

For transaction between bus ' $m$ ' (seller) and bus ' $n$ ' (buyer), the deviation in power exchange can be replaced at the position of seller and buyer as expressed by Equation (23):

$\Delta P_{m}=+P_{t}, \quad \Delta P_{n}=-P_{t}$

$\Delta P_{i j}=\left[\frac{\partial P_{i j}}{\partial \delta_{2}}, \ldots ., \frac{\partial P_{i j}}{\partial \delta_{n}}, \frac{\partial P_{i j}}{\partial V_{g+1}}, \ldots ., \frac{\partial P_{i j}}{\partial V_{n}}\right][J]^{-1}\left[\begin{array}{c}0 \\ \cdot \\ \cdot \\ +P_{t} \\ 0 \\ \cdot \\ \cdot \\ -P_{t}\end{array}\right]=\mathrm{PTDF}^{*} P_{t}$

where, $[J]$ is the Jacobian matrix.

So, PTDFs for the transaction among one seller to buyer can be represented as given by Equation (24): 


$$
\operatorname{PTDF}_{i j, m n}=\left[\frac{\partial P_{i j}}{\partial \delta_{2}}, \ldots ., \frac{\partial P_{i j}}{\partial \delta_{n}}, \frac{\partial P_{i j}}{\partial V_{g+1}}, \ldots \ldots, \frac{\partial P_{i j}}{\partial V_{n}}\right][J]^{-1}\left[\begin{array}{c}
0 \\
\cdot \\
\cdot \\
+1 \\
0 \\
\cdot \\
\cdot \\
-1
\end{array}\right] .
$$

Jacobians and sensitivity factors for line flow are considered without any assumptions.

For transmission pricing economic dispatch has been done by using Power transfer distribution factors (PTDF) [17, 18]. To achieve the above-mentioned process and methods, Matlab 9.0 has been used for programming. In which Load flow analysis, have been programmed using Newton Raphson method to determine the bus power injection in transmission line and losses occurred in the line. Matlab program is also developed for Power Transfer Distribution Factors, Bialek's power tracing and Transmission pricing using MW-Mile methods.

\section{FLOW CHART FOR TRANSMISSION COSTING}

The Flow Chart in Figure 1 explains the process and methodological framework for the analysis and verification of the proposed application of PTDF method, to determine transmission costing and its comparison with other methods.

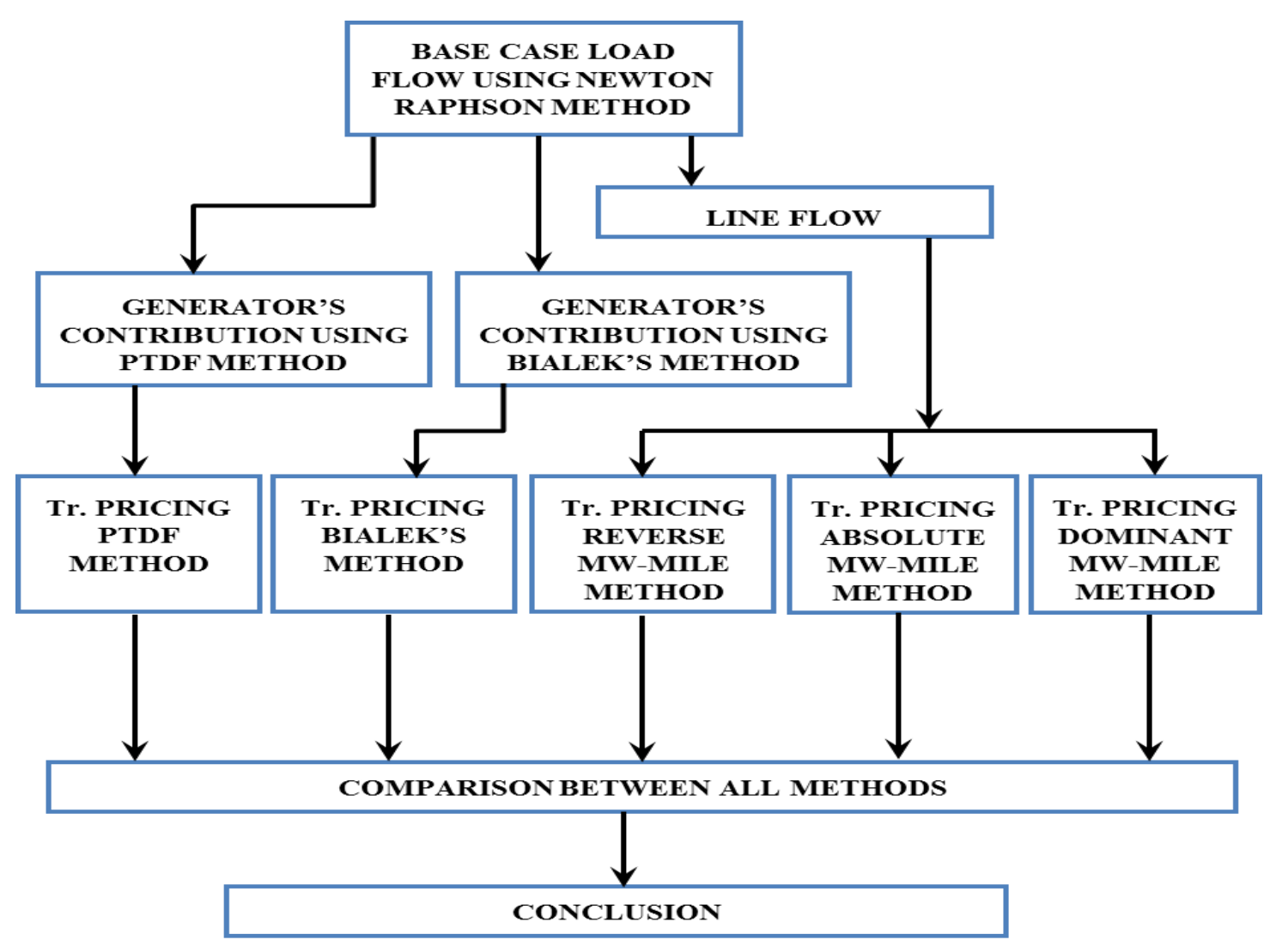

Figure 1. Flow Chart for Transmission Costing 
Under which, the load flow analysis was performed using N-R method. That was then used to determine the generator contribution using proposed application of PTDF and Bialek's method which was further used to determine the transmission pricing using proposed application of PTDF method and Bialek's method. Also line flow is utilized for determining the MW-mile transmission pricing for Reverse, Absolute and Dominant case. After determination of transmission pricing the comparison has been done and that is further concluded according to the application. This comparison and conclusion is very much beneficial for the operating companies and planning engineers for the selection of method according to the demographic location of the infrastructure.

\section{RESULTS}

IEEE 30- Bus system shown in Figure 2 has been utilized to establish the above stated objective.

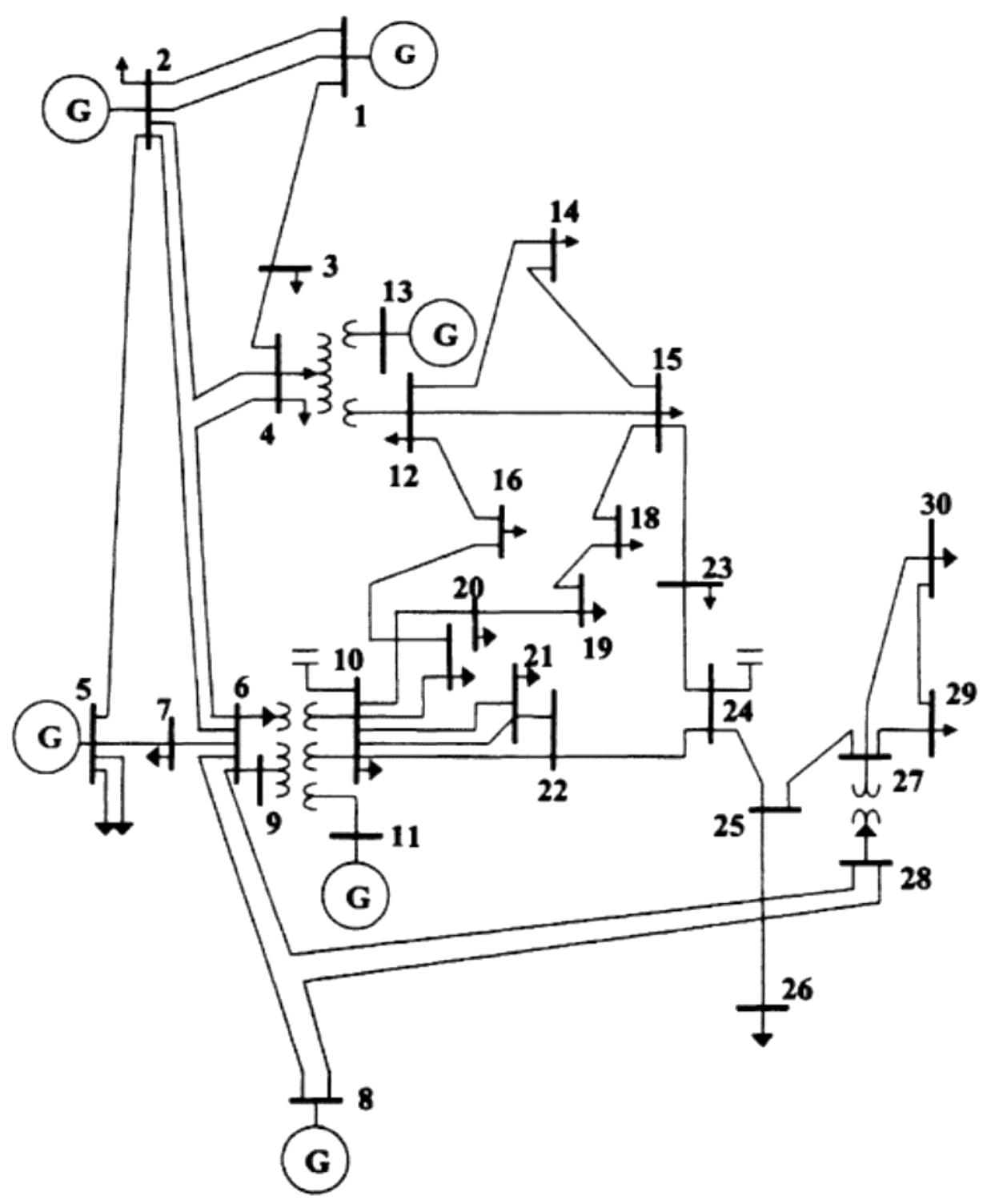

Figure 2. IEEE 30- Bus system 
The outcomes of Transmission Pricing for the IEEE 30-Bus is given below to verify the output of the proposed application of PTDF method with the published results in literature. These results were used as a part of the procedure to calculate Transmission Pricing.

In Table 1, the Newton Raphson load flow analysis results of IEEE 30-Bus are provided, where Power Injected, Power Generated, Voltage Phase Angle and Loads results are evaluated at different nodes of the network. The Voltage is in per unit value and the Phase angles are in degrees. This table shows the amount of power generated by each generator to satisfy the loads at different buses. It also provides the information about the Voltage and Phase angle at the respective buses for the power generated at each bus.

Table 1. Newton Raphson Load Flow analysis

\begin{tabular}{|l|l|l|l|l|l|l|l|l|}
\hline \multirow{2}{*}{ BUS No. } & VOLTAGE & ANGLE & \multicolumn{2}{l}{ POWER INJECTED } & \multicolumn{2}{l|}{ POWER GENERATED } & \multicolumn{2}{l|}{ LOAD } \\
\cline { 2 - 9 } & pu & Degree & MW & MVAR & MW & MVAR & MW & MVAR \\
\hline 1 & 1.06 & 0.00 & 79.72 & 18.78 & 79.72 & 18.78 & 0 & 0 \\
\hline 2 & 1.04 & -1.18 & 39.27 & -8.26 & 60.97 & 4.44 & 21.7 & 12.7 \\
\hline 3 & 1.03 & -3.16 & -2.40 & -1.20 & 0.00 & 0.00 & 2.4 & 1.2 \\
\hline 4 & 1.03 & -3.76 & -7.60 & -1.60 & 0.00 & 0.00 & 7.6 & 1.6 \\
\hline 5 & 1.01 & -3.02 & 0.00 & -20.15 & 0.00 & -1.15 & 0 & 19 \\
\hline 6 & 1.02 & -4.52 & 0.00 & 0.00 & 0.00 & 0.00 & 0 & 0 \\
\hline 7 & 1.00 & -4.45 & -22.80 & -10.90 & 0.00 & 0.00 & 22.8 & 10.9 \\
\hline 8 & 1.02 & -5.16 & -30.00 & 5.41 & 0.00 & 35.41 & 30 & 30 \\
\hline 9 & 1.02 & -7.34 & 0.00 & 0.00 & 0.00 & 0.00 & 0 & 0 \\
\hline 10 & 0.99 & -8.88 & -25.80 & -2.00 & 0.00 & 0.00 & 25.8 & 2 \\
\hline 11 & 1.06 & -7.34 & -0.01 & 20.38 & 0.00 & 20.38 & 0.01 & 0 \\
\hline 12 & 1.03 & -5.89 & -11.20 & -7.50 & 0.00 & 0.00 & 11.2 & 7.5 \\
\hline 13 & 1.06 & -3.18 & 37.00 & 23.26 & 37.00 & 23.26 & 0 & 0 \\
\hline 14 & 1.01 & -6.67 & -6.20 & -1.60 & 0.00 & 0.00 & 6.2 & 1.6 \\
\hline 15 & 1.01 & -6.63 & -8.20 & -2.50 & 0.00 & 0.00 & 8.2 & 2.5 \\
\hline 16 & 1.00 & -8.03 & -3.50 & -1.80 & 0.00 & 0.00 & 3.5 & 1.8 \\
\hline 17 & 0.98 & -9.89 & -40.00 & -5.80 & 0.00 & 0.00 & 40 & 5.8 \\
\hline 18 & 0.99 & -8.14 & -3.20 & -0.90 & 0.00 & 0.00 & 3.2 & 0.9 \\
\hline 19 & 0.98 & -8.85 & -9.50 & -3.40 & 0.00 & 0.00 & 9.5 & 3.4 \\
\hline 20 & 0.98 & -8.92 & -2.20 & -0.70 & 0.00 & 0.00 & 2.2 & 0.7 \\
\hline 21 & 0.99 & -8.41 & -17.50 & -11.20 & 0.00 & 0.00 & 17.5 & 11.2 \\
\hline 22 & 0.99 & -8.10 & 21.59 & 0.00 & 21.59 & 0.00 & 0 & 0 \\
\hline 23 & 1.01 & -5.66 & 16.00 & -1.60 & 19.20 & 0.00 & 3.2 & 1.6 \\
\hline 24 & 0.99 & -6.91 & -8.70 & -6.70 & 0.00 & 0.00 & 8.7 & 6.7 \\
\hline 25 & 1.01 & -5.41 & 0.00 & 0.00 & 0.00 & 0.00 & 0 & 0 \\
\hline 26 & 0.99 & -5.84 & -3.50 & -2.30 & 0.00 & 0.00 & 3.5 & 2.3 \\
\hline 27 & 1.03 & -4.24 & 26.91 & 0.00 & 26.91 & 0.00 & 0 & 0 \\
\hline 28 & 1.02 & -4.64 & 0.00 & 0.00 & 0.00 & 0.00 & 0 & 0 \\
\hline 29 & 1.01 & -5.44 & -2.40 & -0.90 & 0.00 & 0.00 & 2.4 & 0.9 \\
\hline 30 & 1.00 & -6.31 & -10.60 & -1.90 & 0.00 & 0.00 & 10.6 & 1.9 \\
\hline TOTAL & & & 5.18 & -25.08 & 245.39 & 101.12 & 240.21 & 126.20 \\
\hline
\end{tabular}


Table 2. Line Flow and Losses

\begin{tabular}{|c|c|c|c|c|c|c|c|c|c|c|}
\hline FROM & TO & $\mathrm{P}$ & Q & FROM & TO & $\mathrm{P}$ & Q & LINE & LOSS & LINE LIMIT \\
\hline BUS & BUS & MW & MVAR & BUS & BUS & MW & MVAR & MW & MVAR & MW \\
\hline 1 & 2 & 45.25 & 16.64 & 2 & 1 & -44.86 & -15.45 & 0.40 & 1.19 & 130 \\
\hline 1 & 3 & 34.47 & 7.40 & 3 & 1 & -33.97 & -5.35 & 0.50 & 2.05 & 130 \\
\hline 2 & 4 & 28.00 & 0.75 & 4 & 2 & -27.59 & 0.50 & 0.41 & 1.25 & 65 \\
\hline 3 & 4 & 31.57 & 6.78 & 4 & 3 & -31.44 & -6.41 & 0.13 & 0.37 & 130 \\
\hline 2 & 5 & 20.08 & 12.85 & 5 & 2 & -19.83 & -11.82 & 0.25 & 1.04 & 130 \\
\hline 2 & 6 & 36.05 & 2.76 & 6 & 2 & -35.36 & -0.64 & 0.70 & 2.12 & 65 \\
\hline 4 & 6 & 36.05 & 8.49 & 6 & 4 & -35.90 & -7.95 & 0.15 & 0.54 & 90 \\
\hline 5 & 7 & 19.83 & -5.17 & 7 & 5 & -19.64 & 5.64 & 0.19 & 0.48 & 70 \\
\hline 6 & 7 & 3.22 & 14.83 & 7 & 6 & -3.16 & -14.65 & 0.06 & 0.18 & 130 \\
\hline 6 & 8 & 25.55 & -7.20 & 8 & 6 & -25.47 & 7.48 & 0.08 & 0.28 & 32 \\
\hline 6 & 9 & 25.41 & -5.63 & 9 & 6 & \begin{tabular}{|l|}
-25.41 \\
\end{tabular} & 6.96 & 0.00 & 1.32 & 65 \\
\hline 6 & 10 & 14.47 & 3.00 & 10 & 6 & -14.47 & -1.87 & 0.00 & 1.13 & 32 \\
\hline 9 & 11 & 0.01 & -19.63 & 11 & 9 & \begin{tabular}{|l|}
-0.01 \\
\end{tabular} & 20.38 & 0.00 & 0.75 & 65 \\
\hline 9 & 10 & 25.40 & 24.20 & 10 & 9 & -25.40 & -22.93 & 0.00 & 1.27 & 65 \\
\hline 4 & 12 & 15.37 & -1.31 & 12 & 4 & -15.37 & 1.89 & 0.00 & 0.58 & 65 \\
\hline 12 & 13 & -37.00 & \begin{tabular}{|l|}
-20.89 \\
\end{tabular} & 13 & 12 & 37.00 & 23.26 & 0.00 & 2.38 & 65 \\
\hline 12 & 14 & 6.75 & 2.45 & 14 & 12 & -6.69 & -2.33 & 0.06 & 0.12 & 32 \\
\hline 12 & 15 & 13.57 & 6.26 & 15 & 12 & -13.43 & -5.99 & 0.14 & 0.27 & 32 \\
\hline 12 & 16 & 20.85 & 2.79 & 16 & 12 & -20.46 & -1.96 & 0.39 & 0.83 & 32 \\
\hline 14 & 15 & 0.49 & 0.73 & 15 & 14 & \begin{tabular}{|l|}
-0.49 \\
\end{tabular} & -0.73 & 0.00 & 0.00 & 16 \\
\hline 16 & 17 & 16.96 & 0.16 & 17 & 16 & -16.73 & 0.38 & 0.23 & 0.54 & 16 \\
\hline 15 & 18 & 12.83 & 1.17 & 18 & 15 & -12.66 & -0.81 & 0.17 & 0.35 & 16 \\
\hline 18 & 19 & 9.46 & \begin{tabular}{|l|}
-0.09 \\
\end{tabular} & 19 & 18 & -9.40 & 0.20 & 0.06 & 0.12 & 16 \\
\hline 19 & 20 & -0.10 & -3.60 & 20 & 19 & 0.10 & 3.61 & 0.00 & 0.01 & 32 \\
\hline 10 & 20 & 2.33 & 4.36 & 20 & 10 & \begin{tabular}{|l|}
-2.30 \\
\end{tabular} & -4.31 & 0.02 & 0.05 & 32 \\
\hline 10 & 17 & 23.46 & 6.68 & 17 & 10 & -23.27 & \begin{tabular}{|l|}
-6.18 \\
\end{tabular} & 0.19 & 0.50 & 32 \\
\hline 10 & 21 & -5.45 & 11.69 & 21 & 10 & 5.51 & -11.56 & 0.06 & 0.12 & 32 \\
\hline 10 & 22 & -6.26 & 5.91 & 22 & 10 & 6.32 & \begin{tabular}{|l|}
-5.80 \\
\end{tabular} & 0.05 & 0.11 & 32 \\
\hline 21 & 22 & -23.01 & 0.36 & 22 & 21 & 23.07 & -0.24 & 0.06 & 0.12 & 32 \\
\hline 15 & 23 & \begin{tabular}{|l|}
-7.11 \\
\end{tabular} & 3.05 & 23 & 15 & 7.17 & -2.93 & 0.06 & 0.12 & 16 \\
\hline 22 & 24 & -7.80 & 6.04 & 24 & 22 & 7.91 & -5.86 & 0.11 & 0.17 & 16 \\
\hline 23 & 24 & 8.83 & 1.33 & 24 & 23 & \begin{tabular}{|l|}
-8.73 \\
\end{tabular} & -1.12 & 0.10 & 0.21 & 16 \\
\hline 24 & 25 & \begin{tabular}{|l|}
-7.88 \\
\end{tabular} & 0.29 & 25 & 24 & 8.00 & \begin{tabular}{|l|}
-0.08 \\
\end{tabular} & 0.12 & 0.20 & 16 \\
\hline 25 & 26 & 3.54 & 2.37 & 26 & 25 & -3.50 & -2.30 & 0.04 & 0.07 & 16 \\
\hline 25 & 27 & -11.54 & -2.29 & 27 & 25 & 11.69 & 2.57 & 0.15 & 0.28 & 16 \\
\hline 28 & 27 & -1.95 & -2.97 & 27 & 28 & 1.95 & 3.02 & 0.00 & 0.05 & 65 \\
\hline 27 & 29 & 6.19 & 1.66 & 29 & 27 & -6.10 & -1.50 & 0.08 & 0.16 & 16 \\
\hline 27 & 30 & 7.09 & 1.66 & 30 & 27 & -6.93 & -1.36 & 0.16 & 0.30 & 16 \\
\hline 29 & 30 & 3.70 & 0.60 & 30 & 29 & -3.67 & -0.54 & 0.03 & 0.06 & 16 \\
\hline 8 & 28 & -4.53 & 0.62 & 28 & 8 & 4.54 & -0.58 & 0.01 & 0.04 & 32 \\
\hline 6 & 28 & 2.60 & -3.56 & 28 & 6 & -2.60 & 3.57 & 0.00 & 0.01 & 32 \\
\hline \multicolumn{8}{|c|}{ TOTAL LOSS } & 5.18 & 21.75 & \\
\hline
\end{tabular}


Line Flow in the transmission lines for the constrained case is shown in Table 2. Where the line flow is evaluated for both directions of the network in order to determine the Line losses. The Line limit is considered for each line, where it can be seen that Bus 16 to Bus 17 is found to be violating the line flow limit and found to be congested as the power flow in this line is $16.96 \mathrm{MW}$ which is more than the line limit for this line. Which can be further utilized to find the congestion cost of the line.

The total transmission costing using application of PTDF method is shown as a bar chart in Figure 3. Using the generator's contribution obtained from the proposed application of PTDF method transmission costing is evaluated, considering the transmission line cost. The obtained results for total transmission cost using proposed application of PTDF method is 178.26 (\$/MW)

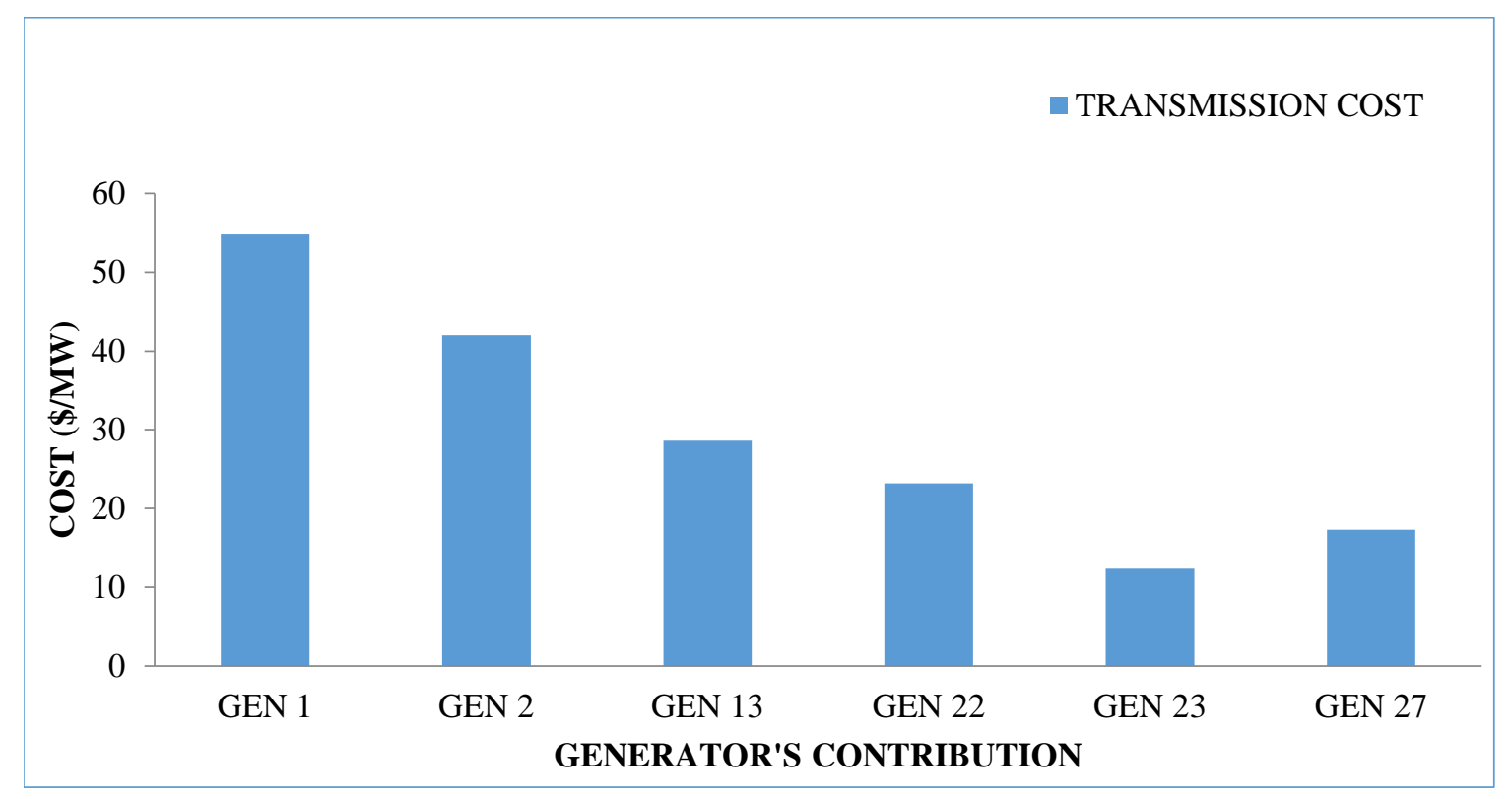

Figure 3. Transmission costing using PTDF method

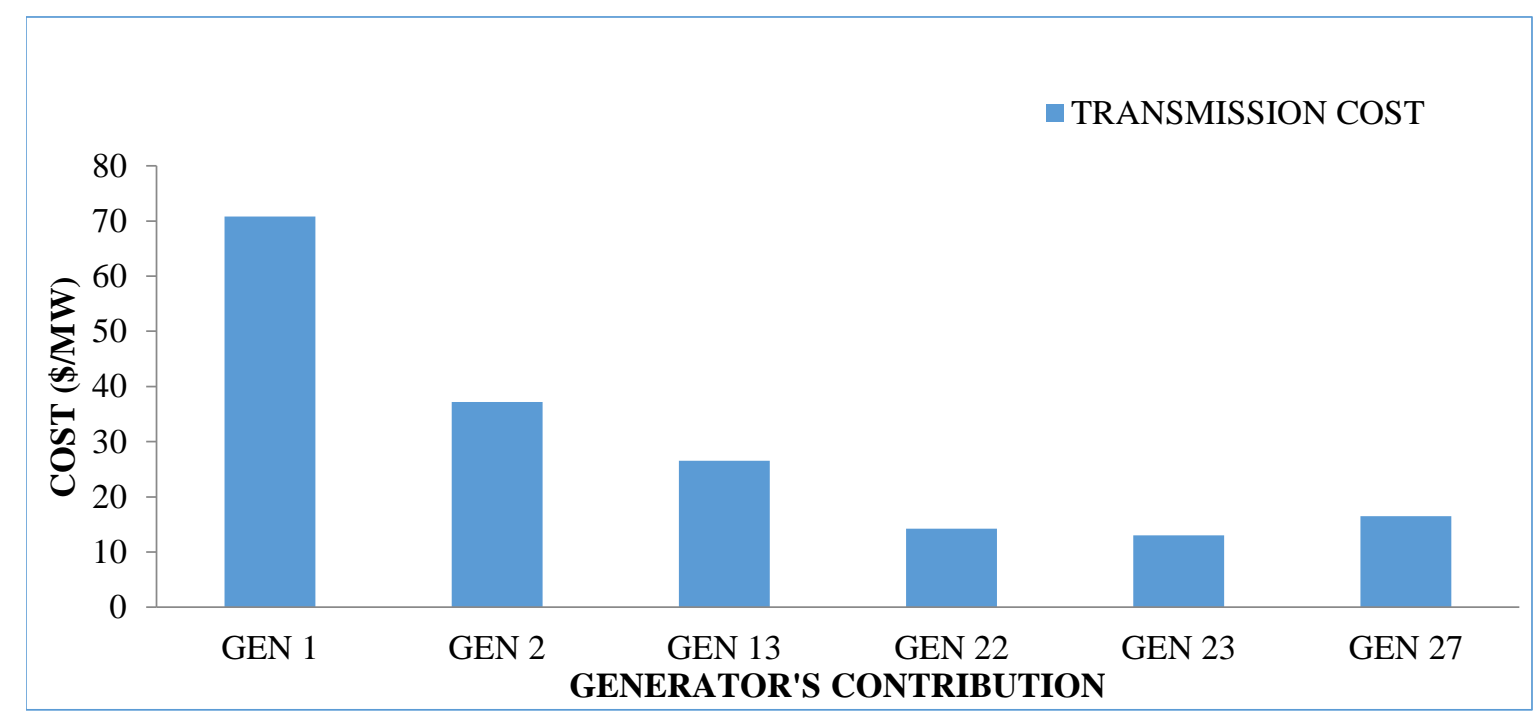

Figure 4. Transmission costing using Bialek's method 
The transmission Costing using Bialek's method is shown as a bar chart in Figure 4. The obtained results for total transmission cost using Bialek's method is $178.26(\$ / \mathrm{MW})$ which is same as the cost obtained using proposed application of PTDF method.

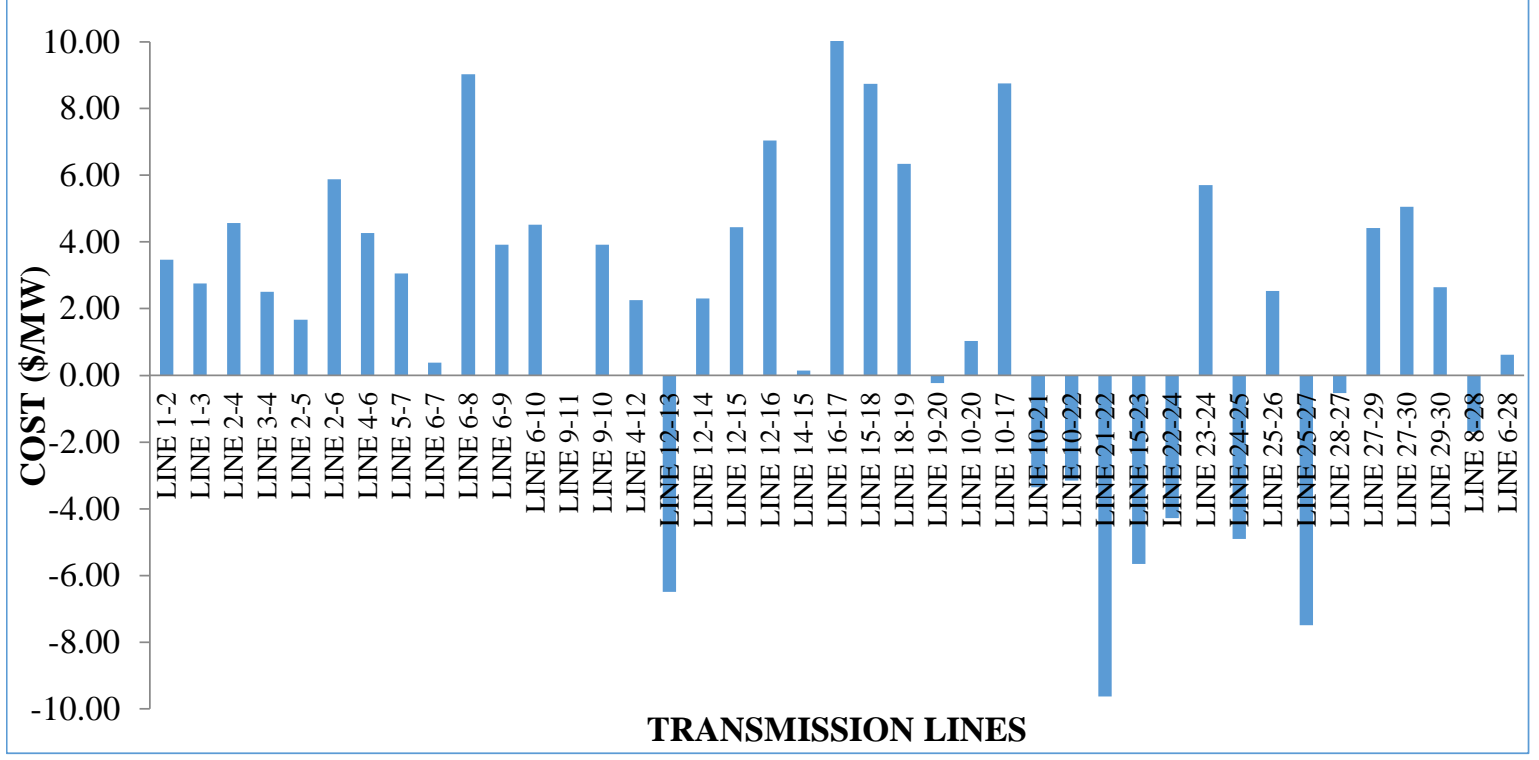

Figure 5. Transmission costing using Reverse MW mile method

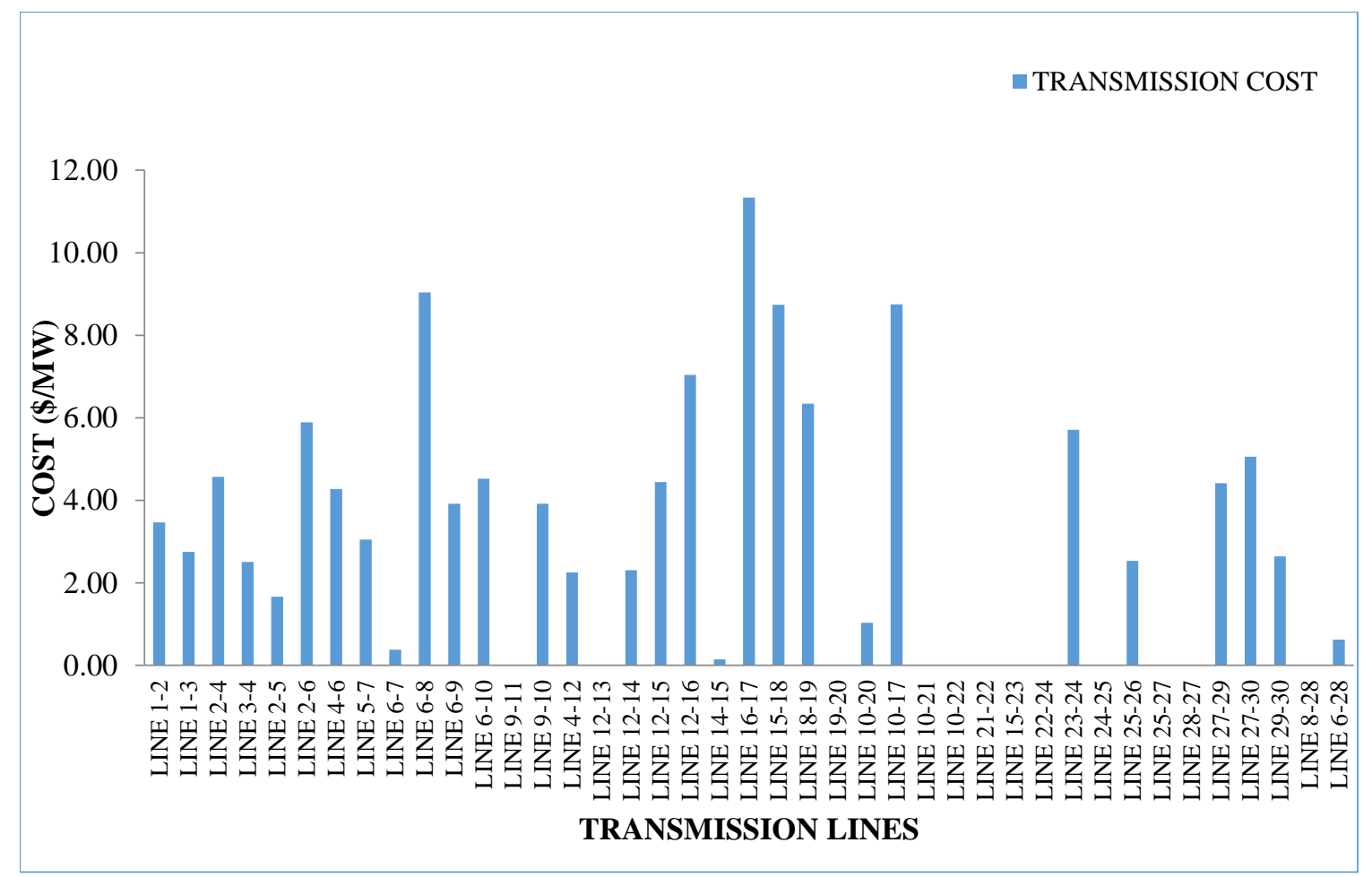

Figure 6. Transmission costing using Dominant MW mile method 


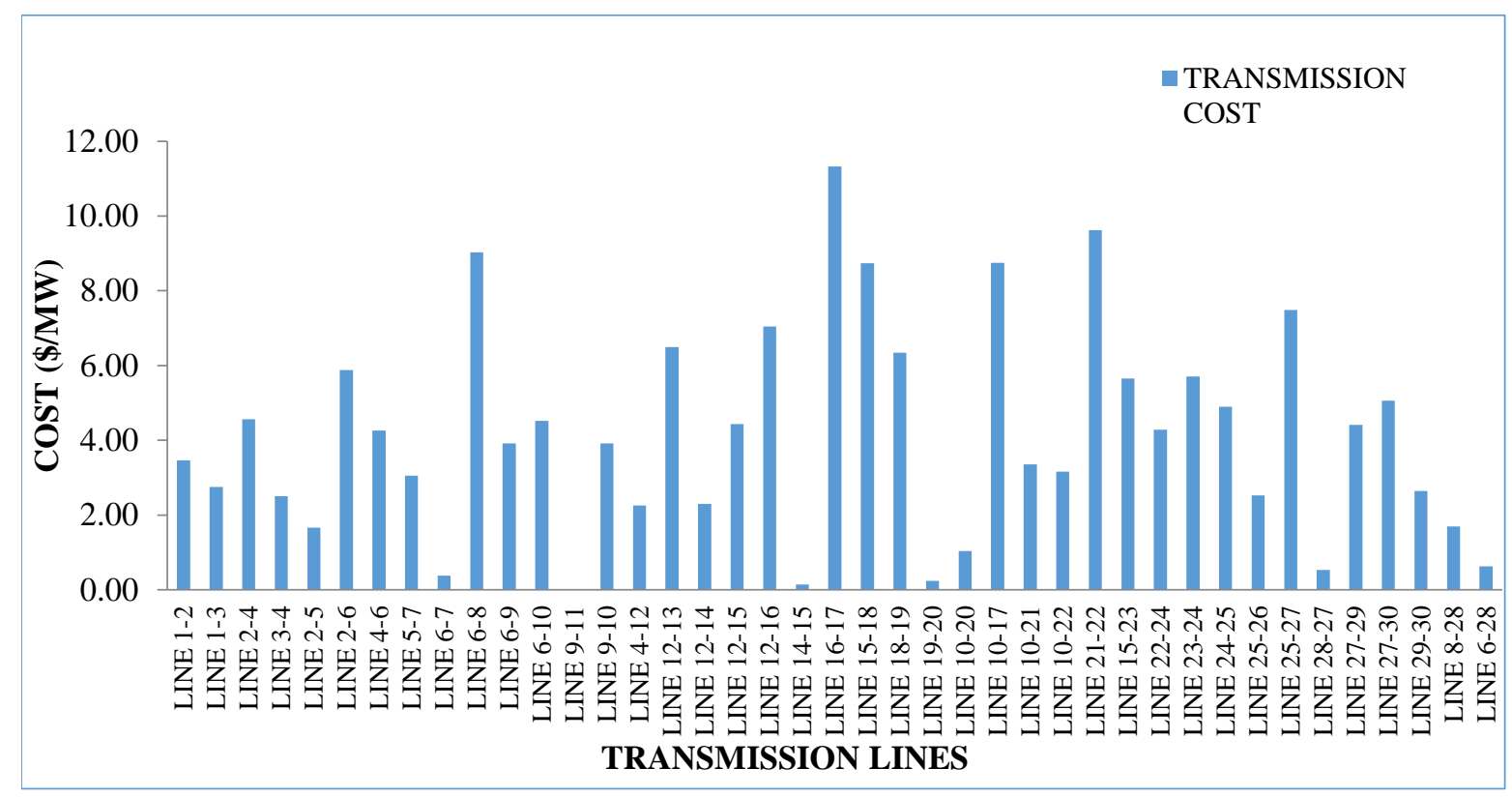

Figure 7. Transmission costing using Absolute MW mile method

Also, the transmission cost using Reverse MW Mile method, Dominant MW Mile method and Absolute MW Mile method are found to be 75.82 (\$/MW), 123.25 (\$/MW) and $170.69(\$ / \mathrm{MW})$, which is shown as a bar chart in Figures 5,6 and 7. The basis of MW mile method is the cost of transmitting power per unit distance, so the evaluation is carried out by considering the contribution of each line for the flow of power.

The negative transmission cost for some transmission line in Figure 5 is due to flow of power in opposite direction to the actual power flow.

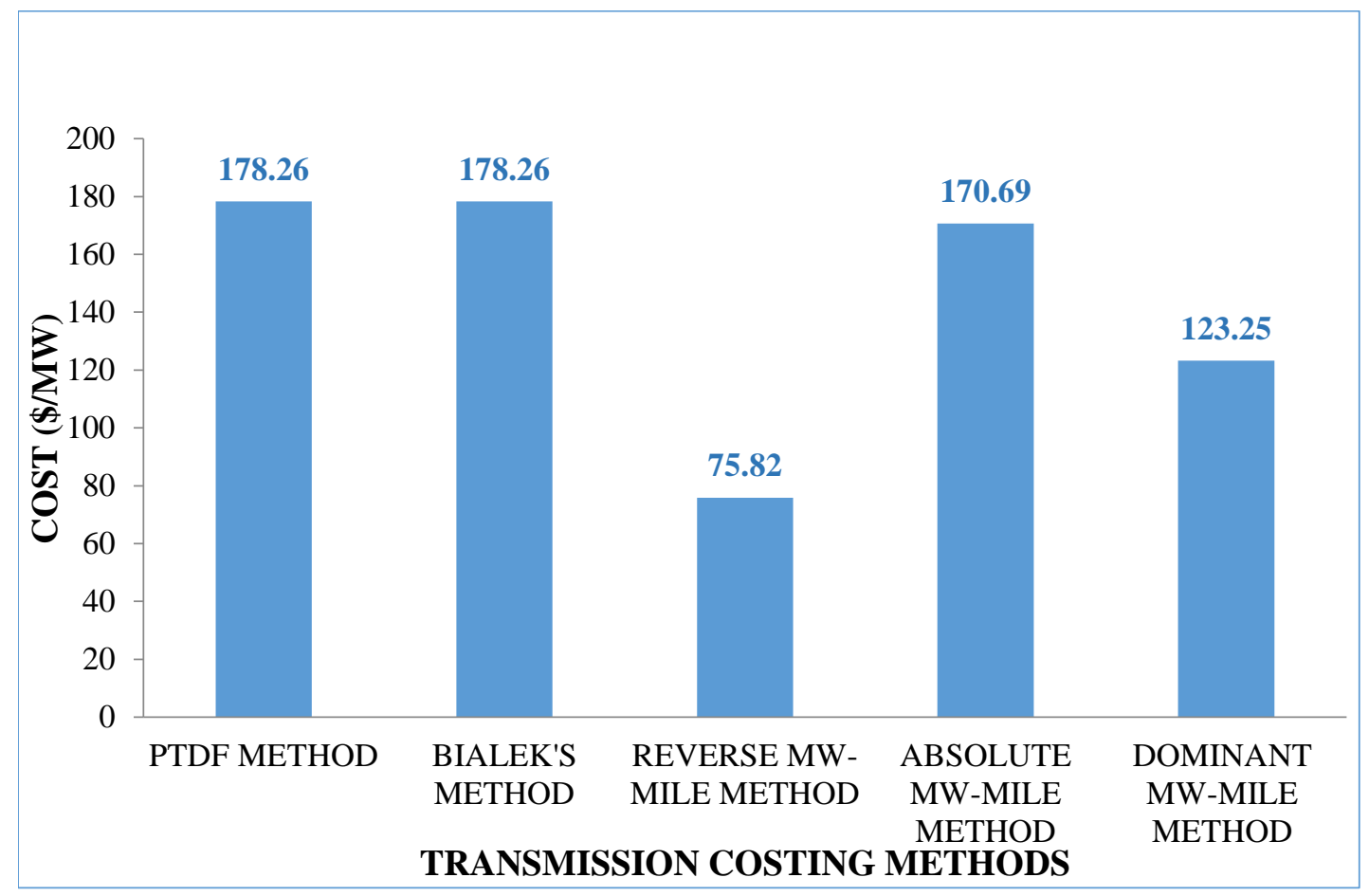

Figure 8. Total transmission cost comparison 
From these results it can be seen that the cost evaluated from Reverse MW mile method is very low as compared to other MW mile methods, but the result obtained from Absolute MW mile method is comparable to the result obtained from proposed application of PTDF method and Bialek's method.

The comparative results for total transmission cost using all the mentioned methods in paper is shown in Figure 8. Where it can be seen that the result obtained by proposed application of PTDF method is 178.26 ( $\$ / \mathrm{MW}$ ), which is to the same as the cost obtained using Bialek's method. Also by comparing the transmission cost using the proposed application of PTDF method with Absolute MW Mile method it gives a similar results i.e.170.69 (\$/MW). This comparison shows that proposed application of PTDF method is justified and can be used for estimating the transmission cost of the transmission system.

After analyzing the cost obtained from all of the results it can be seen that the cost obtained from the proposed application of PTDF method is more than all the mentioned methods, so this method is also justified by obtaining sufficient revenues for the transmission system operators for maintenance and future development/extension of transmission network.

\section{DISCUSSION}

In the IEEE 30-bus system shown in Figure 1 the line length is considered as 100 miles and the power transaction cost is taken as 1000 \$MW-hr-mile-annum.

For transmission pricing evaluation the main process is to generate a load flow solution of the system. Firstly, the load flow evaluation is done using Matlab programming, to determine the Line Flows and their losses. Then using the line flow, PTDF can be determined. PTDF represents the incremental change, power flow in line change due to change in any injected power in any bus. These factors are not dependent of reference bus selection and also independent of any operating conditions

As in the Dominant MW-Mile method the network consumers are being priced for the direct power flow in the line and the reverse power flow is not counted so among all the three methods Dominant MW-Mile method can be somewhat considered for transmission pricing.

Similar to dominant MW-Mile method Bialek's Transmission Pricing method also charge for the direct power flow. Absolute MW-Mile method calculate the prices depending upon the magnitude of the power and ignores the direction of flow so it is not much appropriate for the Transmission Pricing. Reverse MWMile method also considers the reverse power flow in the line and charges accordingly, this method does not recover the total price of the power transmission so it is also not appropriate for the Transmission Pricing

Table 3. Comparison between different Transmission costing methods

\begin{tabular}{|l|l|l|}
\hline \multirow{2}{*}{ TRANSMISSION COSTING METHODS } & IEEE 30 BUS & 7 BUS \\
\cline { 2 - 3 } & COST $(\$ / M W)$ & COST $(\$ / M W)$ \\
\hline PTDF METHOD & 178.26 & 78.73 \\
\hline BIALEK'S METHOD & 178.26 & 59.23 \\
\hline REVERSE MW MILE METHOD & 75.82 & 46.05 \\
\hline ABSOLUTE MW MILE METHOD & 170.69 & 71.34 \\
\hline DOMINANT MW MILE METHOD & 123.25 & 58.69 \\
\hline
\end{tabular}

All the above discussed methods and their process of evaluating the transmission cost can be easily compared as provided in Table 3. In which the comparison between the mentioned and the proposed method transmission cost evaluated is provided for IEEE 30 Bus system and \& bus system. Where, the proposed application of PTDF method takes the consideration of both direct and reverse power flow that is why the 
cost obtained using the proposed method is more than all the compared methods, which can be justifiably applied to find the Transmission pricing that will add to the revenue collection for the transmission operators for future expansion.

\section{CONCLUSION}

The Electric Power Industry combines several methods for allocating transmission costs, reflecting the diverse range of priorities, assumptions and current practice of allocating transmission costs. The costing process must serve the individual as well as collective interests of the stakeholders and also has to be based on laws of physics. Hence a nascent need for an agreed "quantitative analysis and qualitative decisionmaking" framework, to cater for the stakeholder's need to consider mutually accepted priorities, benefits, practical considerations of the complex technical, socio-economic and political costing process.

The investigations in the paper have shown that a combination of methods is common practice, reflecting the diversity of priorities. Even for allocating transmission costs, the international practice shows a pattern of "mixing and matching" elements of the different methods. The proposed framework offers the decision makers in the Industry a wider perspective and costing of electrical power transmission considering constraints and priority assumptions. Further it can also be concluded form the results that PTDF, which are the sensitivity factors and were utilized for power tracing can also be utilized for transmission pricing and other analysis like congestion management. The importance of the proposed evaluation has found to be justified and can be utilized to calculate the Transmission pricing that can generate the reasonable revenue to transmission operators for future expansion. This investigation can also be carried further for reliability benefit analysis of bulk power system and power system security.

\section{CONFLICTS OF INTEREST}

No conflict of interest was declared by the authors.

\section{REFERENCES}

[1] Kumar, N., Das, D. and Pathy, N. P., "Transmission Pricing Reform in India: CERC's Next Frontier. Energy Sources”, Part B: Economics, Planning, and Policy, 8(4): 328-338, (2013).

[2] Orfanos, G.A., G. T. Tziasiou, P. S. Georgilakis, N. D. Hatziargyriou, "Evaluation of Transmission Pricing Methodologies for Pool Based Electricity Markets", PowerTech 2011, IEEE Trondheim: 1$8,(2011)$.

[3] Shilaja, C, Ravi, K., "Multi-Objective Optimal Power Flow Problem using Enhanced Flower Pollination Algorithm", Gazi University Journal of Science, 30 (1): 79-91, (2017).

[4] Ruderer, D. and Zöttl, G., "Transmission pricing and investment incentives", Utilities Policy, 55: 14$30,(2018)$.

[5] Shivaie, M., Kiani-Moghaddam, M. and Ansari, M., "Transmission-service pricing by incorporating load following and correlation factors within a restructured environment", Electric Power Systems Research, 163: 538-546, (2018).

[6] Green, R., "Nodal Pricing of Electricity: How much does it cost to get it wrong?", Journal of Regulatory Economics, 31(2): 125-149, (2007).

[7] Billinton, R. and Jonnavithula, S., "A test system for teaching overall power system reliability assessment", IEEE Transactions on Power Systems, 11(4): 1670-1676, (1996). 
[8] Pan, J., J. Zolezzi, H. Rudnick, F. Danitz, J.W. Bialek, Y. Teklu , S. Rahman, K. Jun "Review of Usage-Based Transmission Cost Allocation Methods under Open Access", IEEE Transactions on Power Systems, 15(4): 1218-1224, (2000).

[9] Bialek, J., "Elimination of Merchandise Surplus due to Spot Pricing of Electricity", IEE ProceedingsGeneration Transmission and Distribution. 144(5): 399-405, (1997).

[10] Alvarado, F.L., "Solving Power Flow Problems with a Matlab Implementation of the Power System Applications Data Dictionary", Systems Sciences, Proceedings of the 32nd Annual Hawaii International Conference, HICSS-32: 7-7, (1999).

[11] Christie, R. D., Wollenberg, B. F. and Wangensteen, I., "Transmission Management in the Deregulated Environment", Proceedings of the IEEE, 88(2): 170-195, (2000).

[12] Kumar, A. and Srivastava, S. C., "Call for Papers ESMO 2003”, IEEE Power Engineering Review, 22 (7): 6-6, (2002).

[13] Kumar, J. and Kumar, A., "ACPTDF for Multi-transactions and ATC Determination in Deregulated Markets”, International Journal of Electrical and Computer Engineering, 1: 71-84, (2011).

[14] Kumar, R., Gupta, S. C. and Khan, B., "Power Transfer Distribution Factor Estimate Using DC Load Flow Method", International Journal of Advanced Electrical and Electronics Engineering, 2(6): 155 159, (2013).

[15] Sookananta, B., "The Transmission Transfer Capability: Terms, Definitions, Calculations and Applications", Khon Kaen University Engineering Journal, 39(3): 311-320, (2012).

[16] Ruiz, P. A., Goldis, E., Rudkevich, A. M., Caramanis, M. C., Philbrick, C. R., \& Foster, J. M., "Security-constrained transmission topology control MILP formulation using sensitivity factors", IEEE Transactions on Power Systems, 32(2): 1597-1605, (2017).

[17] Singh A. K. and Özveren C. S., "Congestion pricing in a deregulated market using A.C. Power Transfer Distribution Factors", 50th International Universities Power Engineering Conference: 1-6, (2015).

[18] Singh R, Singh A. K. and Tyagi T, "Locational marginal pricing calculation using PTDF through load flow and conventional GSF: A comparative study", IEEMA Engineer Infinite Conference: 1-6, (2018). 